\title{
Perfil nutricional de recém-nascidos prematuros internados em uma UTI e UCI neonatal
}

\author{
Nutritional profile of newly-born premature \\ admitted to an ICU and neonatal UCI
}

Geicele Baumhardt Varaschini,, Patrícia Molz, ${ }^{1}$ Camila Schreiner Pereira. ${ }^{1}$ 'Universidade de Santa Cruz do Sul (Unisc), Santa Cruz do Sul, RS, Brasil.

Recebido em: setembro 2014 / Aceito em: janeiro 2014 patricia.molz@gmail.com

\section{RESUMO}

Objetivo: Verificar o estado nutricional e a primeira dieta utilizada em recém-nascidos prematuros internados em uma UTI e UCl neonatal. Método: Foram coletadas as informações de prontuários do momento da internação de 20 recém-nascidos, durante março a maio de 2014. A coleta envolveu o registro da data de nascimento e de internação, idade gestacional ao nascer, medidas de peso, comprimento e perímetro cefálico, doenças associadas à prematuridade e primeira dieta recebida pelos recém-nascidos. Resultados: Quanto à prematuridade dos bebês, 15 foram classificados como prematuridade moderada e os demais como prematuridade extrema. A média de peso dos recém-nascidos foi de 1,86 $\pm 0,63 \mathrm{~kg}$. Quanto ao estado nutricional dos bebês em relação à idade gestacional (peso e comprimento), 13 apresentaram peso adequado e 13 eram pequenos no comprimento; 11 dos recém-nascidos estavam com o perímetro cefálico adequado para a idade gestacional de nascimento. A dieta recebida no momento de internação com maior prevalência neste estudo foi a parenteral $(n=10)$, seguida de nada por via oral. Considerações finais: As classificações de peso encontradas nos recém-nascidos foram: baixa, muito baixa ou muitíssimo baixa, possivelmente, pela prematuridade encontrada (moderada ou extrema). Porém, quando o peso foi classificado de acordo com a idade gestacional, a maioria dos recém-nascidos apresentou peso adequado. Houve uma maior frequência de comprimento abaixo do recomendado para idade gestacional nos prematuros investigados. Conforme a classificação do perímetro cefálico, a maioria estava adequada para a idade gestacional. 0 tipo de dieta recebida com maior prevalência no primeiro momento da internação foi a parenteral.

Palavras-chave: Estado Nutricional; Recém-nascidos, Prematuridade; Dieta.

\section{ABSTRACT}

Objective: To check the nutritional status and first diet in premature newborns admitted to an ICU and Neonatal ICU. Method: admission records information of 20 newborns were collected during March-May 2014. Information collected involved recording the date of birth and hospitalization, gestational age at birth, weight measures, length and head circumference, associated diseases and first diet received by newborns. Results: With regard to newborns prematurity, 15 were classified as moderate and others as extreme premature. The average weight was $1.860 \pm 0.63 \mathrm{~kg}$. Regarding nutritional status, the gestational age (weight and length), 13 were classified as normal weight, 13 were small length; 11 out of newborns had appropriate cephalic perimeter for the gestational age of birth. The diet received at the time of hospital admission with a higher prevalence was parenteral $(n=10)$, then anything orally. Final considerations: weight rating of newborns regardless of gestational age was low, very low or extremely low, possibly due to preterm newborns (moderate or extreme). However, when the weight was classified according to gestational age, most of newborns presented adequate weight. There was an increased frequency of length below the recommended for gestational age in preterm investigated. According to the classification of cephalic perimeter, most were appropriate for gestational age. Type of diet used with higher prevalence in first time after hospitalization was parenteral.

Keywords: Nutritional Status; Newborns; Prematurity; Diet. 


\section{INTRODUÇÃO}

A abordagem nutricional de recém-nascidos pré-termos (RNPT) e de recém-nascidos de muito baixo peso (RNMBP), ao nascer, representa um importante desafio nos dias de hoje ${ }^{1}$. A alimentação e as dificuldades encontradas nessa população podem provocar não só o atraso no seu crescimento, como prolongar o tempo de hospitalização ${ }^{2}$. Recursos tecnológicos associados à disponibilidade de profissionais de saúde qualificados têm aumentado as chances de sobrevivência de neonatos RNPT e RNMBP. No entanto, as dificuldades em relação à alimentação vêm sendo motivo de preocupação em especial quanto à amamentação desses bebês ${ }^{1,2}$. De um modo geral, após o quadro respiratório estabilizado nos RNPT, a prioridade torna-se o aspecto nutricional ${ }^{2}$.

A conduta nutricional é um importante aliado no crescimento e no desenvolvimento do RNPT. O leite humano (LH) é considerado o alimento completo e para o RNPT a sua composição se adapta às suas necessidades; no entanto nem sempre é possível utilizá-lo nesses bebês ${ }^{3}$. Na abordagem do RNMBP, a meta nutricional é alcançar um desenvolvimento pós-natal em uma taxa que se aproxime do crescimento e do ganho de peso intrauterino de um feto normal de mesma idade gestacional (IG), sem produzir deficiências nutricionais, efeitos metabólicos indesejáveis ou toxicidades decorrentes de uma exagerada oferta nutricional ${ }^{4}$.

Nascer prematuramente coloca o recém-nascido (RN) numa categoria de grande risco nutricional; nesse sentido, a alimentação representa um contínuo desafio para os responsáveis pela nutrição do RNPT e $\mathrm{RNBP}^{4}$. Portanto, o objetivo deste estudo foi verificar o estado nutricional e a primeira dieta utilizada em recém-nascidos prematuros internados em uma Unidade de Tratamento Intensivo (UTI) e Unidade de Cuidados Intermediários (UCI) neonatal.

\section{MÉTODO}

Trata-se de um estudo transversal, observacional descritivo, com abordagem qualitativa e quantitativa. Foram estudados e avaliados os prontuários de recém-nascidos prematuros internados na UTI e UCl pertencentes ao Hospital de Caridade e Beneficência (HCB) do Município de Cachoeira do Sul - RS, que autorizou, através de Carta de Concessão, a coleta de dados. O estudo foi aprovado pelo Comitê de Ética em Pesquisa da Universidade de Santa Cruz do Sul (UNISC), sob o número CAEE 25712714.7.0000.5343.

O registro dos dados foi realizado através dos prontuários no momento de internação dos $\mathrm{RN}$, no período de março a maio de 2014, não havendo contato direto com o paciente. A coleta envolveu o registro da data de nascimento e de internação na UTI e UCI, IG ao nascimento (semanas), medidas de peso (g), comprimento $(\mathrm{cm})$ e perímetro cefálico $(\mathrm{PC})(\mathrm{cm})$, doenças associadas à prematuridade e primeira dieta recebida pelos RN.

Para identificação do grau de prematuridade utilizou-se a como referência a idade gestacional de nascimento dos RNPT conforme a classificação de Accioly, Saunders e Lacerda $^{5}$ : a) prematuridade limítrofe: os recém-nascidos entre 37 e 38 semanas pós-concepção; b) prematuridade moderada: os recém-nascidos entre
31 a 36 semanas pós-concepção; e c) Prematuridade extrema: os recém-nascidos entre 24 e 30 semanas pós-concepção.

A avaliação do estado nutricional envolveu a utilização de dois critérios: 1) apenas o peso ao nascer, em que os RNPT foram classificados como: a) recém-nascido de baixo peso ao nascer (RNBP), com peso entre 2500 a 1501 gramas; b) RNMBP, peso entre 1500 a 1000 gramas; e c) recém-nascido de muitíssimo baixo peso (RNMMBP), com peso inferior a 1000 gramas $^{6,7}$; e 2) peso e comprimento em relação à IG, sendo utilizada a curva para prematuros de Babson e Benda ${ }^{8}$, atualizada por Fenton ${ }^{9}$ : a) pequeno para a IG (PIG), abaixo do percentil 10; b) adequado para a IG (AIG), percentil 10 até 90; c) grande para a IG (GIG), acima do percentil 90. Para avaliação do PC seguiu-se a mesma referência de classificação ${ }^{8,9}$. Dados sobre a primeira dieta recebida no momento de internação foram coletados a partir dos prontuários. Os dados obtidos, durante a coleta, foram digitados e avaliados no programa Microsoft Excel (versão 2007).

\section{RESULTADOS}

Foram incluídos, no presente estudo, 20 recém nascidos prematuros sendo que destes, 15 estavam internados na UTI e os demais na $\mathrm{UCI}(n=3)$. Dentre os recém-nascidos prematuros, a maior parte $(n=16)$ foi do sexo feminino. Quanto à prematuridade dos bebês, 15 foram classificados como moderada e 5 como extrema. A média de peso encontrado foi de $1860 \pm 63 \mathrm{~g}$. Segundo a classificação de peso ao nascer, 14 dos recém-nascidos eram RNBP, 4 RNMBP e 2 RNMMBP. Na avaliação do peso conforme a IG de nascimento, 15 foram classificados como AIG; 3 como GIG; e 1 como PIG; destas, todas do sexo feminino. Quanto ao comprimento, 13 foram classificados como PIG e conforme O PC, 11 foram classificados como AIG (Tabela 1). Dos recém-nascidos com peso, comprimento e perímetro cefálico PIG, a maioria eram os prematuros extremos. Dentre os recém-nascidos com prematuridade moderada, o peso e o perímetro cefálico estavam AIG, porém o comprimento PIG.

Tabela 1 - Dados da pesquisa dos prontuários dos recém-nascidos prematuros. Unidade de Tratamento Intensivo (UTI) e Unidade de Cuidados Intermediários (UCI), Hospital de Caridade e Beneficência (HCB). Cachoeira do Sul, RS, 2014.

\begin{tabular}{lccc}
\hline Variáveis & PIG & AIG & GIG \\
& $\mathbf{n}$ & $\mathbf{n}$ & $\mathbf{n}$ \\
\hline Peso $(\mathrm{kg})$ & 4 & 13 & 3 \\
Comprimento $(\mathrm{cm})$ & 13 & 6 & 1 \\
PC $(\mathrm{cm})$ & 7 & 11 & 2 \\
\hline
\end{tabular}

Legenda: PIG: pequeno para a idade gestacional; AlG: adequado para a idade gestacional; GIG: grande para a idade gestacional; PC: perímetro cefálico.

Em relação à primeira dieta oferecida aos RNBP no momento da internação (UTI e UCI), foram respectivamente: terapia nutricional parenteral (TNP) exclusiva $(n=10)$, nada por via oral (NPO) $(n=8)$, somente via oral (VO), através de seringa $(n=1)$ e VO através de seringa associado à amamentação no peito da mãe $(n=1)$. Considerando somente os prematuros da UTI, a maioria 
receberam dieta TNP exclusiva $(n=10)$, seguido de NPO $(n=5)$. Já para os bebês internados na $\mathrm{UCl}$, a prevalência foi de 3 para NPO; somente VO, 1; e VO através de seringa associado à amamentação no peito da mãe, 1 .

De acordo com os prontuários, as patologias associadas à prematuridade que mais predominaram, foram: pré-eclâmpsia grave, descolamento de placenta prematura, bolsa rota, líquido amniótico reduzido, mães sem pré-natal, seguido de outras causas em menor proporção como: gestação gemelar, hemograma infeccioso, hematoma vaginal, gestação tardia com hipotireoidismo, gestação precoce, infecção precoce, e mães que desconheciam a gestação. No que concerne às patologias associadas à prematuridade desses recém-nascidos, 15 apresentaram insuficiência respiratória, 1 infecção neonatal e o restante sem registros de complicações.

\section{DISCUSSÃO}

O cuidado neonatal, especialmente em no RNPT, deve ter ênfase no crescimento e na avaliação cuidadosa das condutas alimentares utilizadas ${ }^{10}$. Conforme Sucena e colaboradores ${ }^{11}$, a maior parte dos RN da UTI neonatal são prematuros, sendo que muitos são extremos e com baixo peso ao nascer. O presente estudo obteve uma prevalência de prematuridade moderada, seguido de prematuridade extrema, semelhante a outro estudo realizado em uma UTI neonatal de um hospital municipal de Vitória da Conquista, Bahia que encontrou um alto percentual de recém-nascidos que se enquadram na prematuridade moderada $(n=95)$ e extrema $(n=22)^{12}$.

Minamisava e colaboradores ${ }^{13}$ constataram que $27 \%$ das crianças recém-nascidas prematuras nasceram com peso inadequado (PIG) e 73\% nasceram com o peso AIG, corroborando com os dados encontrados nesse estudo. Os recém-nascidos PIG apresentam maior mortalidade e dificuldade de adaptação neonatal em curto e longo prazo, mostrando que o déficit de crescimento é uma condição patológica ou de doença, sendo adequado manter certo grau de vigilância ${ }^{4}$. Uma coorte populacional realizado no Rio Grande do Sul mostrou que os recém-nascidos pré-termo tardios (RNPT de 34 - 36 semanas), crescem mais rápido que os recém-nascido a termo nos dois primeiros anos de vida, mas persiste com risco de duas a três vezes maior de falha no crescimento em peso e comprimento. Essa falha de crescimento que acontece nos primeiros anos e pode aumentar o risco de doenças futuras ${ }^{14}$.

Ao contrário de nosso estudo, outros autores têm observado que fetos do sexo masculino têm mais chances de nascimento prematuro ${ }^{15}$. Maia e colaboradores observaram, no município de Cruzeiro do Sul, no Acre, que meninas apresentam uma proporção de baixo peso neonatal cerca de 1,3 vezes maior, quando comparadas aos meninos ${ }^{16}$, semelhante ao nosso estudo, no qual o percentual de baixo peso prevaleceu em meninas.

Os fatores determinantes do crescimento e do desenvolvimento adequados de RNPT ainda são pouco conhecidos. O avanço tecnológico tem possibilitado a sobrevivência cada vez maior de RN com peso de nascimento cada vez menor nas últimas décadas; contudo, o crescimento de $\mathrm{RN}$ continua consideravelmente insuficiente, mantendo-se assim até a vida adulta ${ }^{17}$. A maioria dos prematuros investigados nesse estudo foram classificados como PIG no comprimento. Oliveira e colaboradores ${ }^{4}$ sugerem que recém-nascidos prematuros podem vir a ter problemas com estatura no futuro. Já, Finken e colaboradores ${ }^{18}$ demonstraram a importância do padrão de crescimento pós-natal sobre a altura adulta.

O perímetro cefálico é uma medida que está fortemente ligada ao desenvolvimento cerebral, possibilitando identificar a adequação do desenvolvimento cerebral $^{19}$, em que as primeiras semanas pós-natal podem ser críticas para o crescimento cerebral ${ }^{20}$. No presente estudo, houve uma prevalência mais expressiva de recém-nascidos com perímetro cefálico adequado.

A estratégia nutricional tem sido amplamente apontada como fator determinante no tratamento do neonatal, gerando melhora no ganho ponderal de peso e no controle de infecções ${ }^{17}$. No presente estudo, o leite materno (LM) ordenhado exclusivo praticamente não foi recebido pelos bebês, apenas um bebê recebeu LM exclusivo. Scochi e colaboradores ${ }^{21}$ afirmaram que $64,8 \%$ dos bebês internados em uma UTI neonatal de um hospital amigo da criança de Ribeirão Preto-SP, de 1990 a 1996, receberam o LM como primeiro alimento. Em um estudo mais recente, realizado na mesma instituição, esse índice aumentou para $85 \%$, entretanto $60 \%$ da ingestão LM foi associado ao leite artificial. Diferentemente do nosso estudo, no qual a TNP prevaleceu em 50\% ( $n=10)$ dos bebês, Accioly, Saunders e Lacerda ${ }^{4}$ observaram a TNP introduzida precocemente, bem como observaram que a terapia nutricional enteral (TNE) com um volume adequado reduziu o déficit de crescimento pós-natal. É necessário um suporte nutricional especializado em $\mathrm{RN}$ de alto risco para prevenir o retardo de crescimento no primeiro ano de vida.

Martins e colaboradores ${ }^{22}$ evidenciaram a utilização de todas as vias de alimentação na evolução do estado clínico para os RNBP, durante a internação na UTI neonatal. Prevaleceram $100 \%$ dos RNBPs em uso do leite artificial, mas $86,4 \%$ necessitaram permanecer algum período em NPO. De acordo com os autores, no momento da internação na UTI neonatal, é comum que os bebês não recebam alimentação, pois o seu início depende da evolução clínica, sendo recomendável que os RN recebam LM o mais precocemente possível, pois atendem às necessidades proteicas energéticas e imunológicas.

Considerando-se que a etiologia dos aspectos obstétricos da prematuridade é multifatorial; ao se comparar o presente trabalho ao estudo de Rocha e colaboradoes $^{23}$, foi possível perceber em comum nos dois estudos que, de acordo com as patologias associadas das mães e a prematuridade, a doença hipertensiva que ocasiona a pré-eclâmpsia teve grande relevância. Condições socioeconômicas adversas, desnutrição, anemia ferropriva materna, infecções vaginais e do trato urinário, primiparidade jovem e doença hipertensiva na gravidez são intercorrências que contribuem para ocorrência de partos pré-termo e de RNBP ${ }^{23}$. Em um estudo foi constatado que $69,7 \%$ das mães, com quadro de pré-eclâmpsia tiveram filhos com peso inferior a $2500 \mathrm{~g}$, reafirmando a influência na incidência do baixo peso ao nascer, visto que em apenas $30 \%$ dos casos, os nascimentos eram prematuros ${ }^{24}$. Para a Sociedade Australiana de Estudo de Hipertensos na Gravidez, cerca de $25 \%$ dos filhos de mães com pré-eclâmpsia são 
pequenos para a $\mathrm{IG}^{25}$.

Entre as consequências da prematuridade nos RNPT investigados nesse estudo, houve grande frequência de insuficiência respiratória 15 (75\%), similar a outros estudos. Na pesquisa de Bonamigo e colaboradores $^{26}$, os neonatos que possuíam IG inferior a 30 semanas também apresentavam um maior número $(72,7 \%)$ de intercorrências respiratórias. Derish e Frankel $^{27}$ relataram que a insuficiência respiratória é o diagnóstico primário em $50 \%$ das crianças internadas em UTI, principalmente de prematuros.

\section{CONSIDERAÇÕES FINAIS}

A classificação de peso dos recém-nascidos, sem considerar a IG, ficou como baixo, muito baixo ou muitíssimo baixo, possivelmente pela prematuridade encontrada (moderada ou extrema); porém, quando classificado de acordo com a IG, a maioria estava adequada. Conforme a classificação do PC, a maioria estava adequada para a IG, entretanto, houve uma prevalência mais acentuada de comprimento abaixo do recomendado para IG nos prematuros investigados. O tipo de dieta recebida com maior prevalência no primeiro momento da internação foi a parenteral. Compreende-se ainda, que são necessários estudos para identificar o perfil nutricional de recém-nascidos prematuros.

\section{REFERÊNCIAS}

1. Silva RV, Silva IA. A vivência de mães de recém-nascidos prematuros no processo de lactação e amamentação. Esc Anna Nery Rev Enferm, 2009; 13: 108-115.

2. Figueiredo H, Barreiros J, Gonçalves I, Cunha M. A intervenção oral na alimentação do recém-nascido pré-termo de muito baixo peso. Faculdade de Motricidade Humana, 2007; 1: 239-49.

3. Feferbaum R, Quintal VS, Araújo, MCK. Nutrição enteral do recém-nascido de baixo peso. In: Feferbaum R, Falcão MC. Nutrição do recém-nascido. São Paulo: Atheneu; 2005.

4. Oliveira AG, Siqueira PP, Abreu LC. Cuidados nutricionais no recém-nascido de muito baixo peso. Rev Bras Crescimento Desenvolv Hum, 2008; 18:148-54.

5. Accioly E, Saunders C, Lacerda E. Nutrição em obstetrícia e pediatria. São Paulo: Artmed; 2003.

6. Gaíva MAM, Gomes MMF. Cuidando do neonato: uma abordagem de Enfermagem. Goiânia: AB; 2003.

7. Pachi, PR. O pré-termo: morbidade, diagnóstico e tratamento. São Paulo: Rocca; 2003.

8. Babson SG, Benda GI. Growth graphs for the clinical assessment of infants of varying gestational age. J Pediatr. 1976; 89: 814-820.

9. Fenton TR. A new growth chart for preterm babies: Babson and Benda's chart updated with recent data and a new format. BMC Pediatr. 2003; 3:1-10.

10. Callegari FP. Estudo comparativo das condutas nutricionais e crescimento de recém-nascidos prematuros internados em UTI neonatal [trabalho de conclusão de curso]. Novo Hamburgo: Centro Universitário Feevale; 2006.

11. Sucena LP, Furlan MFFM. Incidência da utilização de leite materno ordenhado em uma Unidade de Terapia Intensiva neonatal e caracterização dos recém-nascidos. Arq Ciênc Saúde, 2008; 15: 82-89.

12. Soares DS, Cunha JXP. Adesão ao pré-natal e prematuridade: análise documental entre recém-nascidos de uma UTI. Revista Saúde e Pesquisa, 2012; 5: 501-507.

13. Minamisawa R. et al. Fatores associados ao baixo peso ao nascer no Estado de Goiás. Revista Eletrônica de Enfermagem, 2004; 6: 336-349.

14. Rugolo LMSS. Manejo do recém-nascido pré-termo tardio: peculiaridades e cuidados especiais. Sociedade Brasileira de Pediatria. 1-13, 2011.

15. Grillo, E. Fatores de risco para nascimentos prematuros e espontâneos na maternidade do Hospital Universitário UFSC [dissertação]. Florianópolis: Universidade Federal de Santa Catarina; 2005.

16. Maia RRP, Souza JMP. Fatores associados ao baixo peso ao nascer em município do norte do Brasil. Rev Bras Crescimento Desenvolvimento Hum, 2010; 20: 735-744.

17. Gianini NM, Vieira AA, Moreira MEL. Avaliação dos fatores associados ao estado nutricional na idade corrigida de termo em recém-nascidos de muito baixo peso. Jornal de Pediatria, 2005; 81: 34-40.

18. Finken MJ, Dekker FW, Zegher F, Wit JM. Long-term height gain of prematurely born children with neonatal growth restraint: parallellism with the growth pattern of short children born small for gestational age. Pediatrics, 2006; 118: 640-643.

19. Muraro LO. Ganho de peso gestacional e estado nutricional do neonato [trabalho de conclusão de curso]. Porto Alegre: Universidade Federal do Rio Grande do Sul; 2013.

20. Heird WC. The importance of early nutritional management of low-birth weight infants. Pediatr Rev, 1999; 20: 43-41.

21. Scochi CGS, Ferreira FY, Góes FSN, Fujinaga Cl, Ferecini GM, Leite AM. Alimentação láctea e prevalência do aleitamento materno em prematuros durante internação em um hospital amigo da criança de Ribeirão Preto - SP, Brasil. Cienc Cuid Saúde, 2008; 7: 145-154.

22. Martins EL, Padoin SMM, Rodrigues AP, Zuge SS, Paula CC, Trojahn TC. Alimentação de crianças que nasceram com baixo peso no primeiro ano de vida. Cienc Cuid Saúde, 2013; 12: 515-521.

23. Rocha RCL, Souza E, Guazzelli CAF, Chambô Filho A, Soares EP, Nogueira ES. Prematuridade e baixo peso entre recémnascidos de adolescentes primíparas. Rev Bras Ginecol Obstet, 2006; 28: 530-535.

24. Ferraz TR, Neves ET. Fatores de risco para baixo peso ao nascer em maternidades públicas: um estudo transversal. Rev Gaúcha Enferm, 2011; 32: 86-92.

25. Chaim SRP, Oliveira SMJV, Kimura AF. Hipertensão arterial na gestação e condições neonatais ao nascimento. Acta Paul Enferm, 2008; 21: 53-8.

26. Bonamigo ECB, Seidler SLL, Gattermann MJ, Cerentini CM, Sauer EA, Winkelmann ER. Intercorrências clínicas observadas em prematuros internados em uma Unidade de Terapia Intensiva Neonatal de um hospital do interior do Estado do Rio Grande do Sul. Revista Contexto \& Saúde, 2011; 10: 283-90.

27. Derish MT, Frankel LR. Dificuldade e insuficiência respiratórias. In: Behrman RE, Jenson HB. Nelson: Tratado de Pediatria. Rio de Janeiro: Guanabara Koogan Parte, 2002; 13: 226-311. 